\title{
SCIENTIFIC REPRTS OPEN Scale invariance of BMP signaling gradients in zebrafish
}

\author{
Yan Huang ${ }^{1}$ \& David M Umulis ${ }^{1,2}$
}

Received: 11 December 2018

Accepted: 18 March 2019

Published online: 01 April 2019

\begin{abstract}
In both vertebrates and invertebrates, spatial patterning along the Dorsal-ventral (DV) embryonic axis depends on a morphogen gradient of Bone Morphogenetic Protein signaling. Scale invariance of DV patterning by BMPs has been found in both vertebrates and invertebrates, however the mechanisms that regulate gradient scaling remain controversial. To obtain quantitative data that can be used to address core questions of scaling, we introduce a method to tune the size of zebrafish embryos by reducing varying amounts of vegetal yolk. We quantified the BMP signaling gradient in wild-type and perturbed embryos and found that the system scales for reductions in cross-sectional perimeter of up to $30 \%$. Furthermore, we found that the degree of scaling for intraspecies scaling within zebrafish is greater than that between Danioninae species.
\end{abstract}

\begin{abstract}
An important area in developmental biology focuses on identifying mechanisms of robustness to perturbations that organisms face during development. One key variable that persists in many systems is the ability of the patterns to form in proportion to the size of the domain in which they are active. The ability of the system to adapt to different size domains has also gained a lot of interest from physicists and engineers because the biophysical processes thought to be responsible for developmental pattern formation do not automatically account for differences in size and would instead predict a pattern that would not scale. Recent advances have introduced new hypotheses regarding scaling, however in many systems the mechanisms of scaling remain poorly understood ${ }^{1-3}$. In invertebrates, quantitative evidence of scale invariance has been reported in Drosophila in multiple instances. Dynamic scaling of the Decapentaplegic (Dpp) gradient is reported during growth of the wing imaginal disc in Drosophila melanogaster ${ }^{4,5}$. In flies, both interspecies and intraspecies scaling occurs for the anterior-posterior embryonic patterning by Bicoid (Bcd) and for dorsal/ventral embryonic patterning by Dpp ${ }^{6,7}$. Interspecies scaling is observed in patterns of gap and pair-rule gene expression in blastoderm embryos of Lucilia sericata, Drosophila melanogaster, and Drosophila busckii by Bicoid (Bcd) protein ${ }^{7}$. Intraspecies scaling of tissue development along the anterior-posterior axis in Drosophila melanogaster is reported to be traced back to the scaling of anterior Bcd production rate with embryo volume ${ }^{8}$. These examples of scaling have also provided new insight on mathematical modeling of pattern formation and scaling 6 .
\end{abstract}

In vertebrates, the phenomenon of intraspecies scaling has been reported in skin pattern formation in fish pigmentation, and under bisection in Xenopus and goldfish embryo development but the gradients in these systems have not been quantitatively measured to identify whether the gradients themselves scale or if the downstream correction contributes to the observed scaling ${ }^{10-13}$. Interspecies scaling in neural tube patterning between the zebra finch and the chick is suggested as being traced back to the ratio of activating and repressive transcription factors of the morphogen Sonic Hedgehog $(\mathrm{SHH})^{14}$. In zebrafish, intraspecies scaling of ventral neural tube patterning is reported to be accomplished by the feedback between SHH signaling and Scube2, a component necessary for $\mathrm{SHH}$ release ${ }^{15}$.

Our focus herein is to quantify dorsal/ventral scaling by measuring signaling gradients in zebrafish embryos. Embryonic DV patterning is mediated by the extracellular distribution of Bone Morphogenetic Proteins (BMPs). The BMP morphogen gradients are established by a network of extracellular regulators including antagonists that bind and inhibit ligand-receptor interactions and regulate the formation of the gradient in both vertebrates and invertebrates ${ }^{16,17}$. BMP gradient formation has been studied and several potential mechanisms for how the antagonists shape the gradients have been proposed in Drosophila ${ }^{18-24}$, Xenopus ${ }^{13,25,26}$, Nematostella ${ }^{27}$, zebrafish ${ }^{16,28,29}$ and mouse $\mathrm{e}^{30}$.

To determine whether DV patterning by BMPs in zebrafish is scale invariant, we developed a protocol to physically reduce the size of embryos. We measured the BMP signaling gradient using quantitative imaging and we measured the degree of scaling in zebrafish DV patterning. This not only allows us to identify the scale invariance

\footnotetext{
${ }^{1}$ Agricultural and Biological Engineering, Purdue University, West Lafayette, IN, 47907, USA. ${ }^{2}$ Weldon School of Biomedical Engineering, Purdue University, West Lafayette, IN, 47907, USA. Correspondence and requests for materials should be addressed to D.M.U. (email: dumulis@purdue.edu)
} 


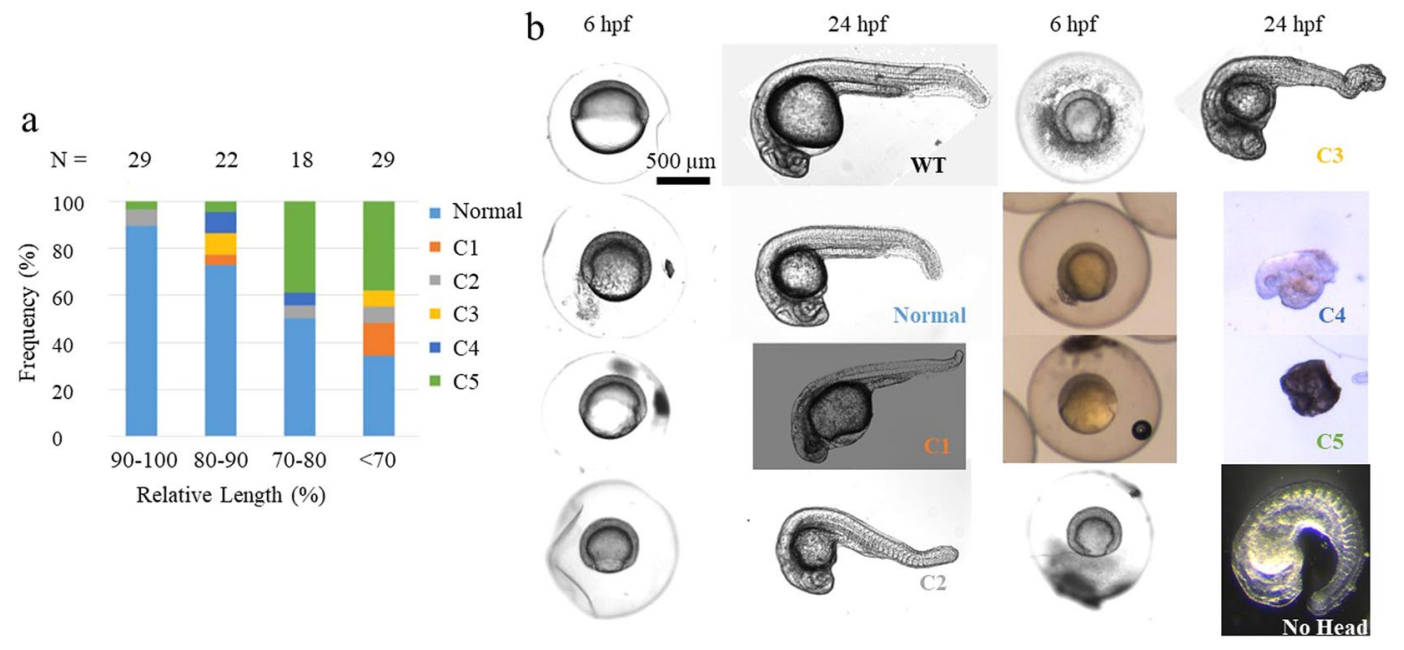

Figure 1. Phenotype distributions of cut embryos. (a) The bar plot shows the frequencies of different phenotypes of Cut larvae at $1 \mathrm{dpf}$. Normal: DV proportions same as wild-type. C1 C5: dorsalized phenotypes from weakest to strongest, with the proportions of DV axis tissues different from WT or some tissues along DV axis missing. The definitions of these 5 classes of phenotypes are used as in Mullins et al. C1: no tail fin. C2: no tail vein. C3: no yolk extension. C4: no tail. C5: the yolk spills out of the embryo and the embryo lyses ${ }^{1}$. The number of embryos that look ventralized is also combined to Class 5 category. (b) Lateral view of live WT (top left two) and Cut embryos at $6 \mathrm{hpf}$ and $24 \mathrm{hpf}$ under bright-field microscope. Embryos displaying normal, C1, C2, C3, C4, C5(the embryo lyses) and ventralized (no head) phenotypes are imaged at 6 hpf and 24 hpf with phenotype labels on the $24 \mathrm{hpf}$ image panel.

phenomenon in zebrafish embryonic DV patterning but also opens a window for us to further investigate the formation and regulation of BMP gradients by perturbing size. In addition to surgical size modifications of sibling Danio rerio, we quantified morphogen gradients and the degree of scaling of DV patterning between zebrafish (Danio rerio) and another closely related species giant danio (Devario aequipinnatus, abbreviated by $\mathrm{Gd}$ ) in the Danioninae clade. Through experiments and quantitative analysis, we found that zebrafish embryos are able to scale for a range of size reductions, however scaling does not persist between $\mathrm{Gd}$ and zebrafish. We provide a new paradigm for discovering scale invariant systems and investigating potential mechanisms, as well as new metrics to quantify the degree of scale invariance and compare scaling among different systems.

\section{Results and Discussion}

Intraspecies scaling of zebrafish embryonic DV patterning. We generated Cut embryos by removing vegetal yolk between the 8-cell and 256-cell cleavage stages, and split the eggs into two groups, one group of eggs was fixed at shield stage for staining, the other group was placed in the incubator to observe the phenotypes at $1 \mathrm{dpf}$. From this experiment, we find a distribution of phenotypes including many that are smaller than WT that display a proportional DV axis the same as WT. Figure 1a plots the frequency of Cut embryos and displays different phenotypes after yolk removal surgery. Over 30\% of the Cut embryos that are 30 50\% smaller than WT display a normal phenotype, and the ratio is higher than $50 \%$ for Cut embryos that have smaller perturbations ranging from $0 \sim 30 \%$ smaller than WT. The distribution of embryos suggests that the system is capable of scaling, however the level of survival decreases as the perturbation increases.

Figure $1 \mathrm{~b}$ shows the normal phenotype and other phenotypes among Cut embryos at $1 \mathrm{dpf}$ (Right) and their corresponding images at $6 \mathrm{hpf}$ (Left). The Cut embryos are fixed at the same time when WT grows to shield stage (6 hpf). Figure $1 \mathrm{~b}$ displays normal, C1, C2, C3, C4, and C5 phenotypes among the Cut embryos. The Cut embryo that displays a normal phenotype at $1 \mathrm{dpf}$ is about $13 \%$ smaller in length and $33 \%$ smaller in volume than WT embryo at $6 \mathrm{hpf}$. This is an example of pattern scale invariance among zebrafish embryos of different sizes. The last embryo in Fig. 1b shows a Cut larva with a ventralized phenotype and no head.

To determine if scaling of the dorsal/ventral gradient of BMP signaling is achieved in early development, we quantified the P-Smad5 gradient in populations of cut embryos. P-Smad5 is a direct readout of BMP signaling commonly used for imaging BMP gradient activity. Figure 2 a shows the result after segmentation that yields an embryo point cloud with P-Smad5 labeling intensity shown by the color heatmap for WT and two Cut embryos. The middle Cut embryo displays a similar P-Smad5 gradient as the WT embryo while the ventral P-Smad5 labeling intensity of the rightmost Cut embryo in Fig. 2a has much lower intensity than that of the WT embryo.

According to Fig. 1, the distribution of phenotypes shows that scaling is not guaranteed, at least for the distribution of sizes within the whole population. Similarly, the BMP signaling pattern by P-Smad5 demonstrates increased embryo-embryo variability after yolk removal. Due to this, we measured scaling properties from two populations of embryos- the complete population- including those without appreciable signaling, and a population filtered to only include embryos that exhibit measurable P-Smad5 signaling. The rules for the filtered population are as follows: 


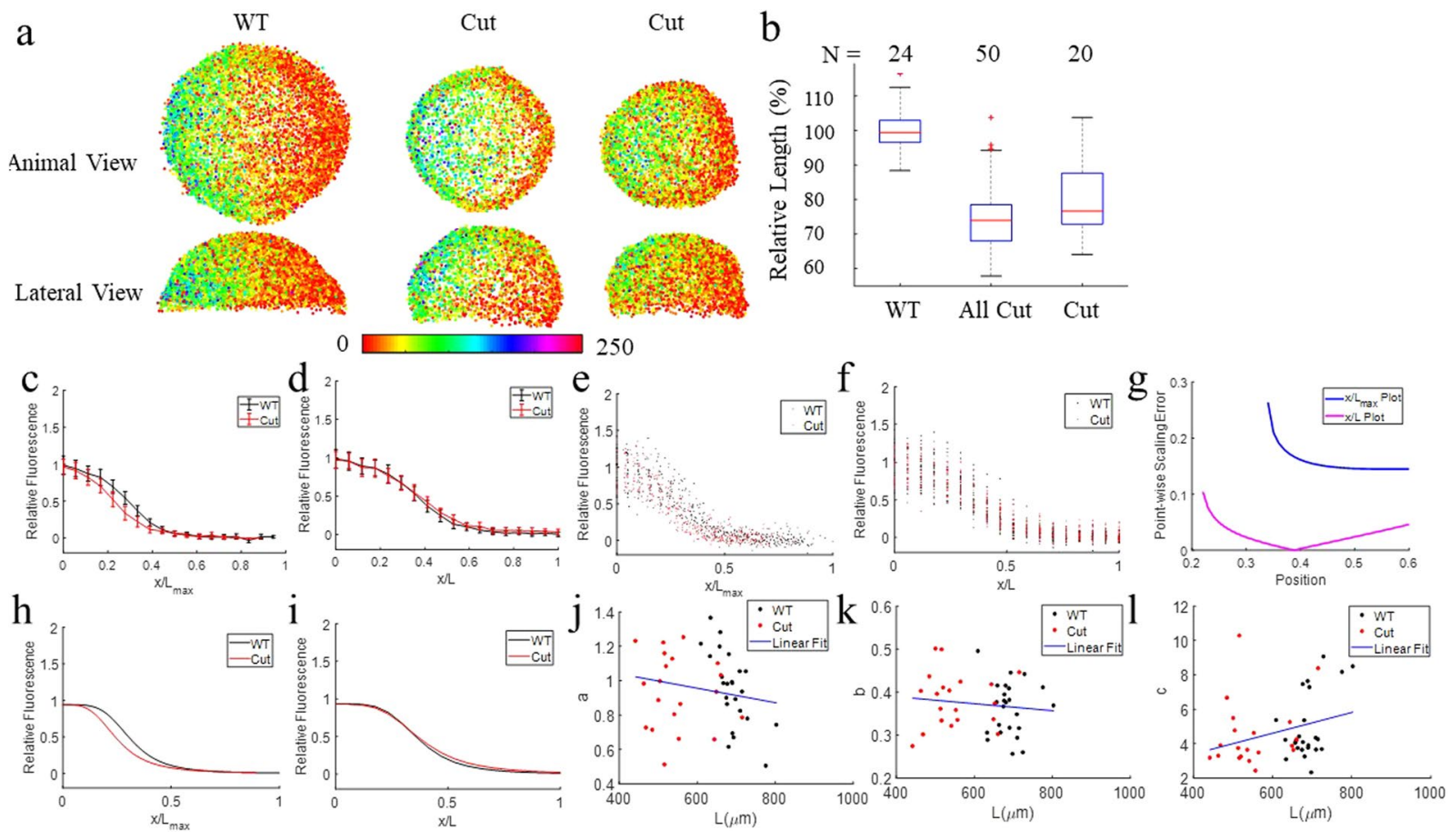

Figure 2. P-Smad5 gradient profiles of WT and Cut scale. (a) The animal and lateral views of P-Smad5 stained $6 \mathrm{hpf}$ embryo point clouds after image processing with P-Smad5 labeling intensity in color. The animal and lateral view of a P-Smad5 stained WT embryo (Left) displays a similar P-Smad5 gradient as the Cut embryo (Middle). The animal and lateral view of a P-Smad5 stained Cut embryo (Right). (b) The boxplot of relative egg sizes of P-Smad5 stained embryos. All Cut are all of the Cut embryos imaged. Cut Compared are the Cut embryos whose difference of P-Smad5 relative intensity between ventral and dorsal half is no less than 0.2 after normalization. From left to right, they are all WT $(\mathrm{N}=24)$, All Cut $(\mathrm{N}=50)$ and Cut Compared (or just 'Cut' for simplication) $(\mathrm{N}=20)$. ( $(\mathbf{c}-\mathbf{f})$ P-Smad5 gradient profiles of WT (black) and Cut (red) embryos after filtration. How $\mathrm{x} / \mathrm{L}_{\max }$ vs $\mathrm{x} / \mathrm{L}$ plots are normalized is described in Materials and Methods, Computational Image Analysis section. (c) Population mean P-Smad5 gradients of WT and Cut after filtering on $\mathrm{x} / \mathrm{L}_{\max }$ plot with variation shown by error bar. (d) Population mean P-Smad5 gradients of WT and Cut on $\mathrm{x} / \mathrm{L}$ plot with variation shown by error bar. (e) P-Smad5 signal of embryos grouped by $10^{\circ}$ interval in dots on $\mathrm{x} / \mathrm{L}_{\max }$ plot, WT in black, Cut Compared in red. (f) P-Smad 5 signal of each embryos averaged by $10^{\circ}$ interval in dots on $\mathrm{x} / \mathrm{L}$ plot, WT in black, Cut Compared in red. Each dot corresponds to an average of P-Smad5 signals of all cells within every $10^{\circ}$ along the margin within one embryo. (g-i) Fitted curves and point-wise SEs for population mean morphogen gradients of Cut and WT on $x / \mathrm{L}$ plot and $\mathrm{x} / \mathrm{L}_{\max }$ plot. (g) SE of Cut and WT population mean morphogen gradients on $\mathrm{x} / \mathrm{L}$ max plot (blue) vs on $\mathrm{x} / \mathrm{L}$ plot (magenta). (h) Curve fitting of population P-Smad5 gradients on $\mathrm{x} / \mathrm{L}_{\max }$ plot. Average P-Smad5 signals of every $10^{\circ}$ along the margin of each WT embryo are fitted with a smooth curve using the Hill equation (equation 1) and plotted as a black line on $\mathrm{x} /$ Lmax plot. (i) Curve fitting of population P-Smad5 gradients on $\mathrm{x} / \mathrm{L}$ plot. Average P-Smad5 signals of every $10^{\circ}$ along the margin of each WT embryo are fitted with a smooth curve using the Hill equation (equation 1 ) and plotted as a black line on $\mathrm{x} / \mathrm{L}$ plot. (j-1): Ratio of curve-fit parameters vs L along the cut and WT embryo margins. (j) Scatter plot of the curve-fit parameter a vsL fitting with a line in blue, used to calculate the scaling power of parameter a. (k) Scatter plot of the curve-fit parameter b vs L fitting with a line in blue, used to calculate the scaling power of parameter b. (l) Scatter plot of the curve-fit parameter $\mathrm{c}$ vs L fitting with a line in blue, used to calculate the scaling power of parameter $\mathrm{c}$.

- Cut embryos need to display endogenous levels of P-Smad5 signaling activity. The Cut embryos with peak P-Smad5 relative intensity beyond the range of the peak P-Smad5 labeling intensity of WT embryos ([0.60, 1.43] after normalization) are discarded.

- Cut embryos need to show signs of a gradient. If the difference of P-Smad5 relative intensity does not exhibit a sufficient gradient between ventral and dorsal half is less than 0.2 after normalization, the Cut embryo is discarded. As a result, in the filtered data-set we retained 20 out of 50 Cut embryos and labeled these embryos 'Cut-Compared'. For simplicity these are referred to as the 'Cut' embryos for the remainder of this work.

Figure $2 \mathrm{~b}$ shows the relative size of fluorescently stained WT and Cut embryos. Most of the Cut embryos are $20 \sim 30 \%$ smaller than WT embryos in length, and 50 70\% smaller in volume. The size variation within WT embryos is about $20 \%$. The Cut embryos are $20 \%$ smaller than WT on average and the smallest Cut embryo is $50 \%$ smaller than WT. Sample size of WT and Cut are 24 and 50 respectively. 


\begin{tabular}{|l|l|}
\hline & p value \\
\hline$a_{W T} v s a_{c u t}$ & 0.820 \\
\hline$b_{W T} v s b_{c u t}$ & 0.30 \\
\hline$c_{W T} v s c_{c u t}$ & 0.44 \\
\hline
\end{tabular}

Table 1. $t$ test of curve-fit parameters of Cut vs WT on $\mathrm{x} / \mathrm{L}$ plot.

\begin{tabular}{|l|l|l|}
\hline & Correlation Coefficient & p value \\
\hline a vs L & -0.18 & 0.24 \\
\hline b vs L & -0.12 & 0.44 \\
\hline c vs L & 0.29 & 0.059 \\
\hline
\end{tabular}

Table 2. Correlation Test for curve-fit parameters vs L.

Metric 1: Morphogen gradients on "scaled" and "relative" position plots. To compare samples and fit them to equivalent functions for statistical comparison we normalize position in two ways. If the gradients scale, then the individual measurements of the gradients would overlap on a normalized $\mathrm{x}$-axis where every gradient is plotted vs "scaled" position $\xi=x / L$ where $x$ is the absolute position and $L$ is the length of each individual and every gradient is on a $[0,1]$ interval. These same gradients, when plotted in absolute position, or any scaling that preserves the relative size of each embryo intact would spread out and not overlap well. On the contrary, if the gradients do not scale and instead have the same absolute gradients, then P-Smad5 would not overlap when plotted against $\xi=x / L$ but they would overlap when plotted versus absolute position or any axis that preserves the relative sizes of the individuals. Therefore, we plot all the gradients for WT and Cut embryos using the "scaled" axis $\xi=x / L$ and also on the "relative" axis where all gradients are normalized to the single longest embryo length $\left(x / L_{\max }\right)$. The "relative" position plots preserve the size differences of the individuals. Normalizing is necessary for our functions for the comparison of the gradients that are developed later herein. Figure $2 \mathrm{c}-\mathrm{f}$ plots P-Smad5 gradients of WT and Cut embryos. Compared to P-Smad5 gradients of Cut and WT embryos on the relative position plot (Fig. 2c), the P-Smad5 gradients of WT and Cut embryos converge better on the scaled $\xi=x / L$ plot (Fig. 2d). Figure $2 \mathrm{f}$ also shows a better overlap of the individuals than the overlap on an $\mathrm{x} / L_{\max }$ plot (Fig. 2e), especially in the range of 0.2 to 0.4 . A student's t-test was used between WT and Cut P-Smad5 relative fluorescence at each node for the 9 ventral lateral space nodes on the $\xi=x / L$ plot and on the $\mathrm{x} / L_{\max }$ plot. The null hypothesis for each test is that the P-Smad5 gradients of WT and Cut embryos have no difference. The $\mathrm{p}$ values of the $\mathrm{t}$ test for WT and Cut P-Smad5 labeling intensities at all the 9 space nodes on the $\xi=x / L$ plot are $>0.05$. This indicates that we could not reject the null hypothesis. In contrast, the p-value for the t-test is below 0.05 at 8 out of 9 space nodes on $\mathrm{x} / L_{\max }$ plot, indicating the WT and Cut P-Smad5 labeling intensities are significantly different at most of the ventral lateral regions on the $\mathrm{x} / L_{\max }$ plot. This comparison confirms the better overlap of WT and Cut P-Smad5 gradients on the $\mathrm{x} / \mathrm{L}$ plot.

The improvement of overlap between the WT and Cut P-Smad5 gradients on the $\mathrm{x} / \mathrm{L}$ plot as opposed to the $\mathrm{x} / L_{\max }$ plot is a hallmark of scaling. The P-Smad5 gradients of each embryo and the population averages are fit to the Hill equation and quantitatively evaluated for scaling by comparing the morphogen gradient curves of WT and Cut embryos plotted in absolute position vs in relative position (Fig. $2 \mathrm{~g}-\mathrm{l}$ ).

Metric 2: Point-wise scaling error. Figure 2g shows that point-wise scaling error (SE) of fitted curves of population averages between WT and Cut embryos on an $\mathrm{x} / \mathrm{L}$ plot is smaller than that on $x / L_{\max }$ plot (throughout lateral regions), indicating a better overlapping/convergence of the morphogen gradients of WT and Cut on $\mathrm{x} / \mathrm{L}$ plot over $\mathrm{x} / \mathrm{L}_{\max }$ plot. Note, SEs at dorsal and ventral ends are not shown because the curve at these two ends are very flat.

Metric 3: T test of curve parameters. We then compared the shapes of gradient curves by the curve parameters of the fitted P-Smad5 gradient curves of each embryo. As shown by $t$ test results in Table 1, all three parameters of the function that fit morphogen gradients of WT and Cut embryos on $\mathrm{x} / \mathrm{L}$ plot do not significantly differ. This indicates that the shape and amplitude of morphogen gradient curves of WT and Cut embryos do not differ. This is consistent with Pearson's correlation test results for curve-fit parameters in Table 2 and the slope of curve parameters vs L in Fig. $2 \mathrm{j}-\mathrm{l}$, that curve-fit parameters do not correlates with size significantly, indicating that the shape and amplitude of curves that fit morphogen gradients on the $\mathrm{x} / \mathrm{L}$ plot do not significantly change with egg size.

The results from the 3 metrics and the normal phenotypes of Cut embryos indicates that the scaling of DV patterning can be traced back to the scaling of morphogen gradients in zebrafish.

Interspecies scaling between zebrafish and giant danio embryonic DV patterning. Giant danio (Gd) and Danio rerio zebrafish are closely related species. They belong to in the same family of Cyprinidae and subfamily of Danioninae. Specifically, the zebrafish line we use to compare with Gd is Tuebingen (Tu). Gd eggs are, on average, $20 \%$ larger and 1.5 3 times larger as adults than zebrafish. As shown in Fig. 3a,b, we observed that the egg size of giant danio is bigger than that of zebrafish. To determine the level of pattern likeness and scaling, 


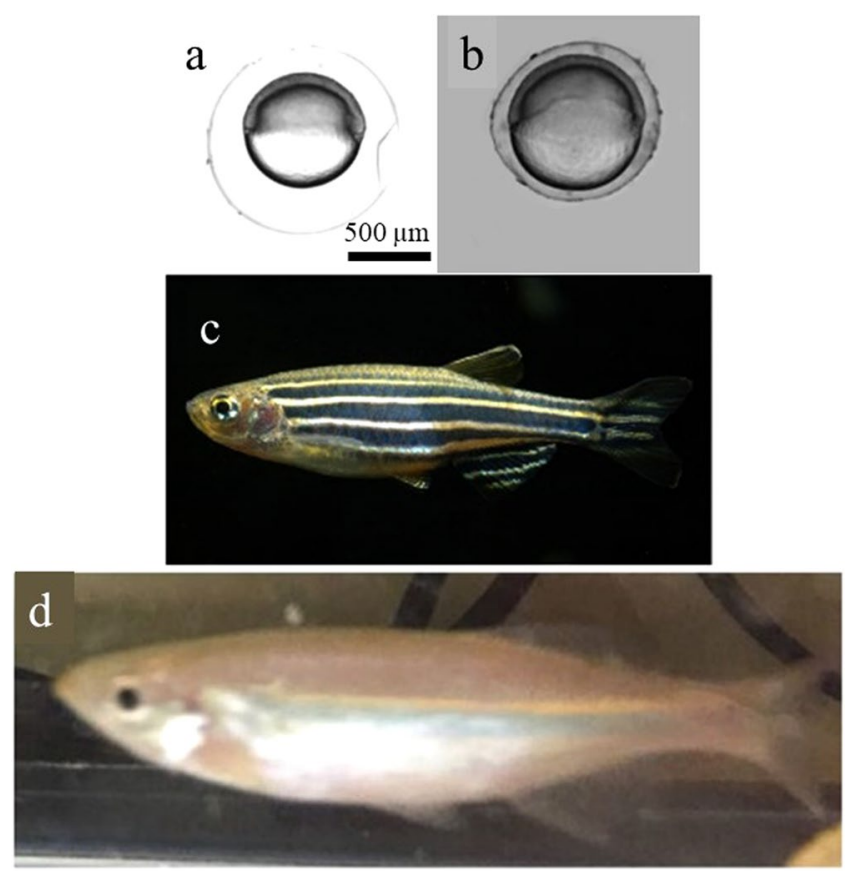

Figure 3. The Gd embryos are significantly bigger than Tu. (a) Bright-field image of one Tu embryo at shield stage. (b) Bright-field image of one Gd embryo at shield stage. (c) Bright-field image of one Tu adult. (d) Brightfield image of one Gd adult.

we measured P-Smad5 morphogen gradients of both and quantified the degree of scaling of their morphogen gradients using the metrics described above.

Metric 1: Morphogen gradients on "scaled" and "relative" position plots. We quantified the P-Smad5 gradient from fluorescence images of the P-Smad5 stained Tu and Gd embryos. Figure 4a shows an example point cloud of Tu and Gd embryos with P-Smad5 labeling intensity in color. We measured P-Smad5 morphogen gradients of $55 \mathrm{Tu}$ and $15 \mathrm{Gd}$ embryos and the average size of Gd is about $24 \%$ larger than that of Tu (Fig. 4b). The P-Smad5 gradients of Tu and Gd embryos exhibit less overlap on the $\mathrm{x} / \mathrm{L}$ plot (Fig. 4d) than they do on the $\mathrm{x} / L_{\max }$ plot (Fig. 4c). This indicates that morphogen gradients of Tu and Gd do not scale. To further evaluate scaling, the P-Smad5 gradients were fit to the same function as before and compared.

Metric 2: Point-wise scaling error (SE). Figure $4 \mathrm{~g}$ shows that point-wise SE of fitted curves of population average between $\mathrm{Tu}$ and $\mathrm{Gd}$ on $\mathrm{x} / \mathrm{L}$ plot is bigger than that on $\mathrm{x} / L_{\max }$ plot (throughout lateral regions), consistently indicates less overlapping of the morphogen gradients of Tu and $\mathrm{Gd}$ on the $\mathrm{x} / \mathrm{L}$ plot as opposed to the $\mathrm{x} / \mathrm{Lmax}$ plot.

Metric 3: T test of curve parameters. We also compared the shapes of gradient curves by the curve parameters, the amplitude parameter $a$, and the slope parameters $b$ and $c$. In Table 3 , the $p$ value of $b$ is less than 0.05 . To achieve power as high as 0.8 for the $t$ test of parameter $b$ given the observed mean and variance of data, the sample size for each group should be at least 9. Here, sample size of Tu and Gd embryos are 55 and 15 respectively, both are higher than 9. Power analysis is conducted for the t test of parameter b given sample size as 15 . The observed power for the $t$ test of parameter $b$ is 0.95 , very high. This means that the parameter corresponding to position shift of the curve, $\mathrm{b}$, of Gd and Tu morphpogen gradients on the $\mathrm{x} / \mathrm{L}$ plot is significantly different by t-test, indicating that the shapes of morphogen gradients of Tu and Gd are significantly different and that they do not scale. This is consistent with the Pearson's correlation test results for the curve-fit parameters in Table 4 and the slope of the curve-fitting parameters vs L in Fig. $4 \mathrm{j}-1$.

The above metrics consistently demonstrate that the DV patterning and corresponding morphogen gradients of Gd embryos do not appear to scale with zebrafish embryos.

\section{Discussion}

The experimental data for Cut and WT zebrafish embryo images and quantitative analysis of the morphogen gradients leads to the conclusion that zebrafish embryos possess some degree of scaling with egg size. This data is consistent with the observed phenotypes- and the distribution of disrupted patterns is consistent with distribution of phenotypes. The analysis herein does not identify the mechanisms that lead to scale invariance, however future studies that couple these imaging approaches, genetic perturbations of BMP pathway components and mathematical modeling will be used to identify these mechanisms.

In contrast to the results of embryonic scaling in surgically perturbed populations of Danio rerio, the P-Smad5 gradient does not appear to scale between closely related species. Gd embryos are significantly bigger than the Tu line of Danio rerio and the morphogen gradients do not scale. The gradients exhibit greater overlap when plotted 

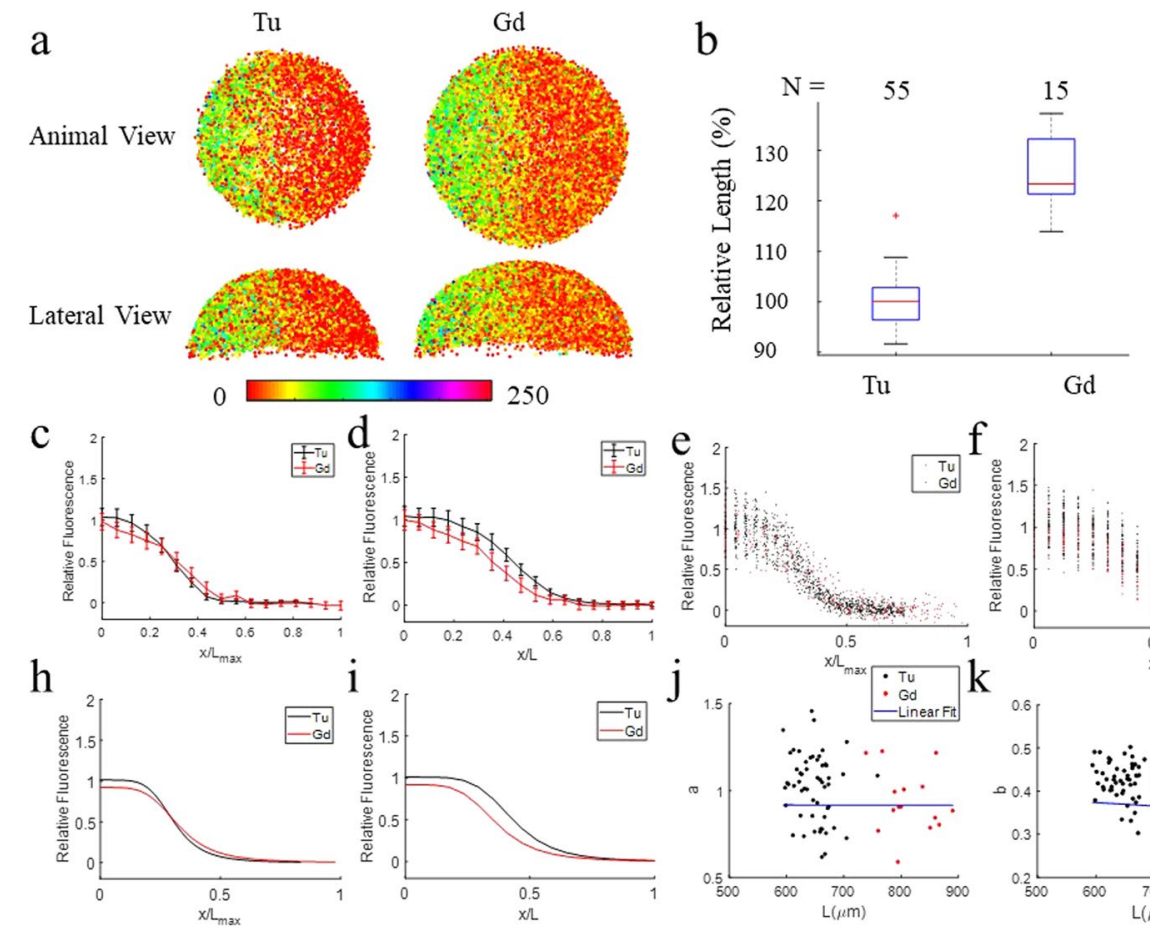

Gd

Figure 4. The P-Smad5 gradient profiles of Gd and Tu do not scale. (a) The animal and lateral views of P-Smad5 stained $6 \mathrm{hpf}$ embryo point clouds of Tu and Gd after image processing with P-Smad5 labeling intensity in color. (b) The distribution of the relative vegetal margin radius length of Tu and Gd. (c-e) P-Smad5 gradient profiles of Gd (red) vs Tu (black) embryos. (c) Population mean P-Smad5 gradients of Gd and Tu on $\mathrm{x} / \mathrm{L}_{\max }$ plot with variation shown by error bars. In each embryo, the P-Smad5 levels of cells are averaged among cells that are nearby within an interval of $\frac{L_{\max }}{18}$ in length. P-Smad5 labeling intensities of all embryos of the same group at the same absolute position are then averaged to plot the errorbar on each position node. (d) Population mean P-Smad5 gradients of $\mathrm{Gd}$ and Tu on $\mathrm{x} / \mathrm{L}$ plot with variation shown by error bars. (e) P-Smad5 signal of each embryos grouped by $10^{\circ}$ interval in dots on $\mathrm{x} / \mathrm{L}_{\max }$ plot, Tu in black, Gd in red. (f) P-Smad5 signal of each embryos averaged by $10^{\circ}$ interval in dots on $\mathrm{x} / \mathrm{L}$ plot, Tu in black, Gd in red. Each dot corresponds to an average of P-Smad5 signals of all cells within every $10^{\circ}$ along the margin within one embryo. (g-i) Point-wise SEs for population mean morphogen gradients of $\mathrm{Gd}$ and Tu after curve fitting on $\mathrm{x} / \mathrm{L}$ plot and $\mathrm{x} / \mathrm{L}_{\max }$ plot. (g) SE of Gd and Tu population mean morphogen gradients on $\mathrm{x} / \mathrm{L}_{\max }$ plot (blue) vs on $\mathrm{x} / \mathrm{L}$ plot (magenta). (h) Average P-Smad5 signals of every $10^{\circ}$ along the margin of each Tu embryo are fitted with a smooth curve using the Hill equation (equation 1) and plotted in black line on $\mathrm{x} / \mathrm{L}_{\max }$ plot. The smooth curve that fits the individual average P-Smad5 signals of Gd embryos are plotted in red line. (i) Curve fitting of population P-Smad5 gradients on $\mathrm{x} / \mathrm{L}$ plot. (j-l) Ratio of curve-fit parameters vs L along the Gd and Tu embryo margins. (j) Scatter plot of the curvefit parameter a vs L fitting with a line in blue, used to calculate the scaling power of parameter $a$. (k) Scatter plot of the curve-fit parameter $b$ vs L fitting with a line in blue, used to calculate the scaling power of parameter b. (l) Scatter plot of the curve-fit parameter c vs L fitting with a line in blue, used to calculate the scaling power of parameter $\mathrm{c}$.

\begin{tabular}{|l|l|}
\hline & p value \\
\hline$a_{T u} v s a_{G d}$ & 0.1369 \\
\hline$b_{T u} v s b_{G d}$ & $2.0746 \mathrm{e}-5$ \\
\hline$c_{T u} v s c_{G d}$ & 0.4689 \\
\hline
\end{tabular}

Table 3. T test of curve-fit parameters of Tu vs Gd for $\mathrm{x} / \mathrm{L}$ plot.

\begin{tabular}{|l|l|l|}
\hline & Correlation Coefficient & p value \\
\hline a vs L & -0.2336 & 0.0516 \\
\hline b vs L & -0.4127 & $3.8431 \mathrm{e}-4$ \\
\hline c vs L & -0.0314 & 0.7963 \\
\hline
\end{tabular}

Table 4. Correlation Test for curve-fit parameters vs L. 
on absolute coordinates or when each is normalized to the maximum sized individual within each population $\left(\mathrm{x} / L_{\max }\right)$.

The next challenge in the study of scale invariance is to move from describing the patterns of scaling relationships to understanding the underlying processes that create the patterns. In the future, possible mechanisms of intraspecies scaling can be investigated. The fact that BMP gradients in Gd and Tu do not scale may have to do with the time it takes for BMP gradient formation and morphogen spread. The time-scale for molecular diffusion is proportional to $\frac{L^{2}}{D}$ (where $D$ is the diffusion coefficient) ${ }^{28}$. We know that the average circumference length the of Gd embryo is about $20 \%$ longer than that of Tu. So, if the values of the diffusion coefficient are about the same in $\mathrm{Gd}$ and $\mathrm{Tu}$, the time for BMP to diffuse across the field of cells increases by about $44 \%$ on average. Furthermore, if the decay rates and production rates of extracellular regulators are about the same in Gd and Tu, the BMP gradient will be shorter as more ligand will decay before it has time to diffuse the greater distances around the embryo. Similarly, since the Tu Cut embryos are about $23 \%$ smaller than Tu WT on average, we can estimate that the decrease in time for diffusion is approximately $41 \%$ on average.

In summary, this work describes our methodological advancements in the study of scaling in zebrafish embryos, and communicates our insights on DV pattern scale invariance within and between fish species. We conclude that DV pattern scale invariance in zebrafish embryos can be traced back to the intraspecies scaling of BMP gradients, and there is no scaling of BMP gradients between zebrafish and giant danio.

\section{Materials and Methods}

Embryonic surgery and confocal fluorescence imaging. Vegetal yolk removal surgery. Embryo size is reduced by removing vegetal yolk during the 8-cell to 256-cell cleavage stage at room temperature. Maternally provided dorsal determinants are known to activate zygotic gene expression cascades required for the formation of the dorsal organizer ${ }^{31-34}$. At 20 minute-post-fertilization (mpf), a microtubule array forms and Syntabulin transports the dorsal determinants to the plus end of microtubules. Around the 2-cell stage (60 mpf), Syntabulin is not detectable by immunostaining and whole embryo lysate immunoprecipitation, indicating the degradation of Syntabulin and the release of the dorsal determinants into cells that are eventually incorporated by dorsal blastomeres $^{35}$. Therefore, the surgery is conducted after the 4-cell stage in order to not disrupt the delivery of dorsal determinants from yolk to cells. Chorion protects the embryo from being destroyed by surface tensions and is not removed during the yolk removal surgery. To do the surgery, we hold a tungsten needle $(0.125 \mathrm{~mm}$ ultra-fine) and pierce across the chorion without destroying it while stabilizing the embryo by holding another part of the chorion with forceps. Then we poke a hole on the vegetal yolk with the needle. The surgery is conducted in IX E3 medium in petri dishes bedded with $1 \%$ agar. The egg size is controlled by removing various amounts of yolk. Yolk removed embryos are called "Cut" while controls that do not have any treatment are called wild-type (WT).

Phenotype observation. Embryos grow in an incubator at $28^{\circ} \mathrm{C}$ for 24 hours after the surgery. In the $1 \mathrm{X}$ E3 solution, $10 \mathrm{U} / \mathrm{ml}$ Penicillin and Streptomycin are added to protect embryos from bacterial infection and Methylene Blue is added to prevent from fungus growth. Images of the embryos are taken under bright-field microscope at shield stage and 1 day-post-fertilization (dpf) to observe the morphological phenotype along the DV axis.

Imaging of P-Smad1/5. Embryos are fixed at shield stage with $4 \%$ paraformaldehyde at $4{ }^{\circ} \mathrm{C}$, blocked in NCS-PBST (10\% fetal bovine serum, 1\% DMSO, 0.1\% Tween 20 in PBS), and probed overnight with a 1:100 dilution of anti-phosphoSmad1/5/9 antibody (Cell Signaling Technology, \#13820 s), followed by a 1:500 dilution of goat anti-rabbit Alexa Fluor 6470 conjugated antibody (Thermo Fisher Scientific, Rockford, IL; Cat\# A-21244, RRID: AB_2535812). Nuclei are stained by sytox orange. Embryos were mounted in BABB (benzyl alcohol (Sigma B-1042) and benzyl benzoate (Sigma B-6630), 1:2 ratio) and scanned using a Zeiss LSM 800 confocal microscope with a $20 \mathrm{X}$ water lens. The detailed protocol of immunostaining and imaging has been published ${ }^{27}$. Here, P-Smad5 fluorescence intensity is used as a readout of extracellular BMP in zebrafish.

Quantification of morphogen gradients. Computational image analysis. We quantified the P-Smad5 gradient through an image analysis pipeline that includes cell segmentation, preprocessing and image registration, and data extraction along the embryo $\operatorname{margin}^{29}$. The marginal circumference of the embryo image is measured by extracting a margin region with a thickness of $60 \mu \mathrm{m}$ and averaging the circumference of the shape. The measurement of embryo size here is half of the margin circumference, denoted as L. In $\mathrm{x} / \mathrm{L}$ plot of each embryo, the position on the horizontal axis is normalized as the position $\mathrm{x}$ on embryo margin divided by this individual embryo's half circumference, L. Note, $L_{m a x}$ is the half margin circumference of the largest embryos among all WT and Cut embryos for intraspecies scaling and among all Tu and Gd embryos for interspecies scaling. In $x / L_{\max }$ plot of each embryo, the position on the horizontal axis is normalized as the position $\mathrm{x}$ on embryo margin divided by the half circumference of the biggest embryo, $L_{\max }$. It is in the same range as the $\mathrm{x}$-axis of a relative position plot $\mathrm{x} / \mathrm{L},[0,1]$ to compare quantities calculated from these two plots.

Data normalization and data reduction. P-Smad5 gradient normalization for populations of images taken on different days are conducted using WT controls ${ }^{27,36}$. P-Smad5 gradients of Gd embryos are normalized within the population of Gd and not normalized against Danio rerio due to possible differences in antibody binding efficiency. Maxima of population averages for Danio rerio and Gd are both set to 1.

Some embryos are too severely damaged by the tungsten needle and do not progress through development. Embryos that are too damaged or exhibit no P-Smad5 signaling due to development arrest are eliminated from the population for quantification of P-Smad5 signaling. 
Curve fitting morphogen gradients. Multiple measures of scaling are used to compare the gradients pointwise measurements for local intensity differences and parameters from functions that are fit to the data. Previous work for morphogen gradient quantification relied on comparison of the length scale of the gradient determined by fitting the data to an exponential function. These functions do not apply here because the gradient shape is not exponential. Many mathematical functions including polynomial, exponential, Fourier, Gaussian, etc. were tested and we found that a variant of the Hill equation fits the shape of morphogen gradients best $\left(R^{2}>0.95\right)$ and was selected to fit the data:

$$
m=\frac{a}{\left(1+\frac{z^{c}}{b}\right)}
$$

$\mathrm{m}$ is the concentration of morphogen and $\mathrm{z}$ is the position, either relative position $\xi\left(\xi=\frac{x}{L}\right)$ or the absolute position scaled to $[0,1]$ by $\frac{x}{L_{\max }}$. Parameter $a$ represents the amplitude of the curve while the slope depends on the value of $b$ and $c$, and the curve has a fixed point at $\left(b, \frac{a}{2}\right)$ (Supplementary Fig. S1).

Ethical approval of using animals. The experiments were approved by Purdue Animal Care and Use Committee (PACUC) with Animal Use Qualification Form on file. We confirm that all experiments were performed in accordance with relevant guidelines and regulations.

\section{Data Availability}

All the 3D point cloud images of embryos and the data matlab files for the study of intraspecies and interspecies scaling are stored in a shared folder. The link to it is https://goo.gl/mcxY3R. It is also included in the supplemental files.

\section{References}

1. Waddington, C. H. Canalization of development and the inheritance of acquired characters. Nature. 150, 563-565 (1942).

2. Patel, N. H. \& Lall, S. Precision patterning. Nature. 415, 748-749 (2002).

3. Lander, A. D. Pattern, growth, and control. Cell. 144, 955-969 (2011).

4. Wartlick, O. et al. Dynamics of Dpp Signaling and Proliferation Control. Science. 331(6021), 1154-1159 (2011).

5. Fried, P. \& Iber, D. "Dynamic scaling of morphogen gradients on growing domains". Nat Communications. 5(5077), 1-12 (2014).

6. Umulis, D., Shimmi, O., O'Connor, M. \& Othmer, H. Organism-Scale Modeling of Early Drosophila Patterning via Bone Morphogenetic Proteins. Developmental Cell. 18(2), 260-274 (2010).

7. Gregor, T., Bialek, W., de Ruyter van Steveninck, R. R., Tank, D. W. \& Wieschaus, E. F. Diffusion and scaling during early embryonic pattern formation. PNAS. 102, 18403-18407 (2005).

8. Cheung, D., Miles, C., Kreitman, M. \& Ma, J. Scaling of the Bicoid morphogen gradient by a volume dependent production rate. Development. 138, 2741-2749 (2011).

9. Umulis, D., O'Connor, M. \& Othmer, H. Robustness of embryonic spatial patterning in Drosophila melanogaster. Curr. Top. Dev. Biol. 81, 65-111 (2008).

10. Mizuno, T., Yamaha, E. \& Yamazaki, F. Localized axis determinant in the early cleavage embryo of the goldfish, Carassius auratus. Development Genes and Evolution. 206(6), 389-396 (1997).

11. Tung, T. C. \& Tung, Y. F. Y. The development of egg-fragments, isolated blastomeres and fused eggs in the goldfish. Proceedings of the Zoological Society of London. 114, 46-64 (1944).

12. Spemann, H. \& Mangold, H. Induction of embryonic primordia by implantation of organizers from a different species. 1923. International Journal of Developmental Biology. 45(1), 13-38 (2001).

13. Inomata, H. et al. Scaling of Dorsal-Ventral Patterning by Embryo Size-Dependent Degradation of Spemann's Organizer Signals. Cell. 153(6), 1296-1311 (2013).

14. Uygur, A. et al. Scaling pattern to variations in size during development of the vertebrate neural tube. Developmental Cell. $37(2), 127$ (2016).

15. Collins Z. M. et al. Megason. A Scube2-Shh feedback loop links morphogen release to morphogen signaling to enable scale invariant patterning of the ventral neural tube, https://www.biorxiv.org/content/early/2018/11/13/469239 (2018).

16. Brazil, D. P., Church, R. H., Surae, S., Godson, C. \& Martin, F. BMP signalling: agony and antagony in the family. Trends in Cell Biology. 25, 249-264 (2015).

17. Dutko, J. A. \& Mullins, M. C. SnapShot: BMP signaling in development. Cell. 145, 636 (2011).

18. Eldar, A. et al. Robustness of the BMP morphogen gradient in Drosophila embryonic patterning. Nature. 419, 304-308 (2002).

19. Marque's, G. et al. Production of a DPP activity gradient in the early Drosophila embryo through the opposing actions of the SOG and TLD proteins. Cell. 91, 417-426 (1997).

20. Holley, S. A. et al. The Xenopus dorsalizing factor noggin ventralizes Drosophila embryos by preventing DPP from activating its receptor. Cell. 86, 607-617 (1996).

21. Peluso, C. E., Umulis, D., Kim, Y. J., O'Connor, M. B. \& Serpe, M. Shaping BMP morphogen gradients through enzyme-substrate interactions. Developmental Cell. 21, 375-383 (2011).

22. Shilo, B. Z., Haskel-Ittah, M., Ben-Zvi, D., Schejter, E. D. \& Barkai, N. Creating gradients by morphogen shuttling. Trends in Genetics. 29, 339-347 (2013).

23. Shimmi, O., Umulis, D., Othmer, H. \& O’Connor, M. B. Facilitated transport of a Dpp/Scw heterodimer by Sog/Tsg leads to robust patterning of the Drosophila blastoderm embryo. Cell. 120, 873-886 (2005).

24. Umulis, D. M., Shimmi, O., O'Connor, M. B. \& Othmer, H. G. Organism-scale modeling of early Drosophila patterning via bone morphogenetic proteins. Developmental Cell. 18, 260-274 (2010).

25. Reversade, B. \& De Robertis, E. M. Regulation of ADMP and BMP2/4/7 at opposite embryonic poles generates a self-regulating morphogenetic field. Cell. 123, 1147-1160 (2005).

26. Zhang, Y. T., Lander, A. D. \& Nie, Q. Computational analysis of BMP gradients in dorsal-ventral patterning of the zebrafish embryo. Journal of Theoretical Biology. 248, 579-589 (2007).

27. Genikhovich, G. et al. Axis Patterning by BMPs: Cnidarian Network Reveals Evolutionary Constraints. Cell Rep. 10(10), 1646-1654 (2015).

28. O'Connor, M. B., Umulis, D., Othmer, H. G. \& Blair, S. S. Shaping BMP morphogen gradients in the Drosophila embryo and pupal wing. Development. 133, 183-193 (2006).

29. Zinski, J. et al. Systems biology derived source-sink mechanism of BMP gradient formation. eLIFE. 6, e22199 (2017). 
30. Zakin, L., Chang, E. Y., Plouhinec, J. L. \& De Robertis, E. M. Crossveinless-2 is required for the relocalization of Chordin protein within the vertebral field in mouse embryos. Developmental Biology. 347, 204-215 (2010).

31. Ober, E. A. \& Schulte-Merker, S. Signals from the yolk cell induce mesoderm, neuroectoderm, the trunk organizer, and the notochord in zebrafish. Developmental Biology. 215(2), 167-181 (1999).

32. Jesuthasan, S. \& Strahle, U. Dynamic microtubules and specification of the zebrafish embryonic axis. Current Biology. 7(1), 31-42 (1997).

33. Schneider, S., Steinbeisser, H., Warga, R. M. \& Hausen, P. $\beta$-catenin translocation into nuclei demarcates the dorsalizing centers in frog and fish embryos. Mech. Dev. 57, 191-198 (1996).

34. Mizuno, T. et al. Removal of vegetal yolk causes dorsal deficiencies and impairs dorsal-inducing ability of the yolk cell in zebrafish. Mechanisms of Development. 81(1-2), 51-63 (1999).

35. Nojima, H. et al. Syntabulin, a motor protein linker, controls dorsal determination. Development. 137(6), 923-933 (2010).

36. Huang, Y. \& Umulis, D. Mechanisms and Measurements of Scale Invariance of Morphogen Gradients. Methods in Molecular Biology: Morphogen Gradients (ed. Dubrulle, J.) 215-262 (New York: Humana Press, 2018).

\section{Acknowledgements}

We thank Dr. Pascal Lafontant's group for providing me giant danio embryos. We acknowledge Dr. Mary Mullins, Francesca Tuazon and Dr. Joseph Zinski for help in developing the protocol for reducing the embryo size, Dr. Bobby Madamanchi for offering feedback for the manuscript, Dr. Xu Wang for sharing experiences and suggestions on experiments, and Yi Ren for dechorionating embryos. We thank the NIH and Grant number HD073156 for support.

\section{Author Contributions}

Y.H. conceived the experiments, conducted the experiments and analyzed the results. D.M.U. also conceived the experiments and analyzed results. Y.H. and D.M.U. wrote the main manuscript text and prepared figures. All authors reviewed the manuscript.

\section{Additional Information}

Supplementary information accompanies this paper at https://doi.org/10.1038/s41598-019-41840-8.

Competing Interests: The authors declare no competing interests.

Publisher's note: Springer Nature remains neutral with regard to jurisdictional claims in published maps and institutional affiliations.

(c) (i) Open Access This article is licensed under a Creative Commons Attribution 4.0 International License, which permits use, sharing, adaptation, distribution and reproduction in any medium or format, as long as you give appropriate credit to the original author(s) and the source, provide a link to the Creative Commons license, and indicate if changes were made. The images or other third party material in this article are included in the article's Creative Commons license, unless indicated otherwise in a credit line to the material. If material is not included in the article's Creative Commons license and your intended use is not permitted by statutory regulation or exceeds the permitted use, you will need to obtain permission directly from the copyright holder. To view a copy of this license, visit http://creativecommons.org/licenses/by/4.0/.

(C) The Author(s) 2019 